\title{
Efectos de la deforestación sobre la diversidad y la estructura del ensamblaje de macroinvertebrados en cuatro quebradas Andinas en Colombia
}

\author{
Esnedy Galeano-Rendón ${ }^{1}$ \& Néstor Javier Mancera-Rodríguez ${ }^{2 *}$ \\ 1. Universidad Nacional de Colombia, Sede Medellín, Grupo Ecología y Conservación de Fauna Silvestre, Calle 59A No. 63-20, \\ Medellín, Colombia; emgaleanor@unal.edu.co \\ 2. Universidad Nacional de Colombia, Sede Medellín. Departamento de Ciencias Forestales, Grupo Ecología y Conservación de \\ Fauna Silvestre, Calle 59A No. 63-20, Bloque 20, oficina 211, Medellín, Colombia; njmancer@unal.edu.co \\ * Correspondencia
}

Recibido 17-XI-2017. Corregido 20-III-2018. Aceptado 05-X-2018.

\begin{abstract}
Effects of deforestation on macroinvertebrate diversity and assemblage structure in four Andean creeks in Colombia. The creeks are strongly influenced by their riparian vegetation, and the deforestation affect the aquatic community. The effects of deforestation on taxonomic and functional diversity of aquatic macroinvertebrate assemblage in four Colombian Andean creeks were evaluated. The study included 12 sites, seven of them with arboreal coverage at their margins in forested areas undisturbed by deforestation (reference stations) and the other five without tree cover in deforested areas (altered stations). Four field samplings were carried out between June 2011 and February 2012, and samples of benthic macroinvertebrates were collected using the D-net method with $500 \mu \mathrm{m}$ mesh and manual collection, and different physicochemical variables were measured in situ. The Shannon-Wienner $\left(\mathrm{H}^{\prime}\right)$ and Simpson's index (D) were calculated. One-way analyses of variance (ANOVAs) to each environmental variable, and canonical discriminant analysis (CDA) to establish differences in environmental variables, taxonomic and functional diversity between references and altered stations were applied. 8370 macroinvertebrate individuals corresponding to 80 taxa were collected in the study, with 6758 individuals and 75 taxa in references stations and 1612 individuals and 50 taxa in altered stations. The reference stations had higher values of diversity and the taxa that made major contributions to the variance between reference and altered stations were Thraulodes, Anacroneuria and Rhagovelia, which were associated with the highest values of Index of Andean riparian vegetation Quality (QBR-And), fluvial habitat index (IHF), Biological Monitoring Working Party Colombia BMWP/Col, EPT index (Ephemeroptera, Plecoptera, and Trichoptera) and temperature in reference stations, whereas Leptonema, Baetodes and Mayobaetis were associated with higher $\mathrm{pH}$ and elevation above sea level in altered stations. The main functional feeding groups (FFG) at the reference stations were collectors-gatherers/scrapers CG / SC (27.2\%), scrapers SC (25.4\%) and predators PR (24.4\%), and for altered stations were collectors-filterers CF (27.5\%), collectors-gatherers/scrapers CG / SC (17.4\%) and scrapers SC (15.7\%). The most abundant life habits were clingers Cg (50.5 \%), taxa with three or more habits $(28.7 \%)$ and skaters Sk $(16.1 \%)$, in the reference stations, and clingers $\mathrm{Cg}(68.1 \%)$, taxa with three or more habits (13.7\%) and burrowers $\mathrm{Bu}(5.2 \%)$ in the altered stations. The knowledge of the variables that determine the taxonomic and functional structure of the macroinvertebrate assemblages, and how they respond to impacts such as deforestation, can help to determine actions of protection or restoration of the environmental and ecological conditions of the Andean streams and the riparian habitats.
\end{abstract}

Key words: taxonomic diversity; functional feeding groups; life habits.

Galeano-Rendón, E., \& Mancera-Rodríguez, N. J. (2018). Efectos de la deforestación sobre la diversidad y la estructura del ensamblaje de macroinvertebrados en cuatro quebradas Andinas en Colombia. Revista de Biología Tropical, 66(4), 1721-1740. 
Las quebradas son sistemas abiertos que presentan una fuerte relación con su entorno terrestre (Allan, 2004), razón por la cual los cambios de uso de suelo que remplazan áreas de bosque en sistemas ganaderos y agrícolas afectan las condiciones físicas del hábitat acuático y las comunidades bióticas allí presentes (Giraldo, Chará, Zúñiga, Chará-Serna, \& Pedraza, 2014). Los bosques de ribera intervienen en los procesos que ocurren entre los ecosistemas terrestres y acuáticos, beneficiando aspectos como la retención de nutrientes o las contribuciones de materia orgánica alóctona, al igual que regulan condiciones como la radiación solar, la temperatura, la forma e hidrología del cauce y la deposición de sedimentos finos, que inciden en la composición y estructura de las comunidades acuáticas (e.g., Allan, 2004; Sweeney et al., 2004; Schneider \& Winemiller, 2008; Lorion \& Kennedy, 2009).

La región Andina de Colombia presenta altas tasas de deforestación, debido a causas directas como la ampliación de la frontera agrícola y la conversión a pastos para la ganadería (Armenteras, Cabrera, Rodríguez, \& Retana, 2013), y las áreas de bosque de montaña y de tierras bajas disminuyeron de 11006893 ha en 1985 a 9528961 ha en 2005 (Armenteras, Rodríguez, Retana, \& Morales, 2011). Entre los impactos negativos de esta tendencia se incluye la destrucción de bosques ribereños, lo cual ocasiona pérdida de diversidad taxonómica y funcional de las comunidades biológicas, las cuales responden diferenciadamente de acuerdo a la intensidad y duración de las presiones ambientales o antrópicas a las que se encuentren sometidas (Death \& Winterbourn, 1995; Death, 1996; Mesa, 2010).

Los macroinvertebrados acuáticos dependen del tipo de energía que llega al cauce y se caracterizan por degradar la hojarasca en distintos niveles tróficos, por lo que se consideran sensibles a la disponibilidad de alimento y la cantidad de sustratos que aporta la vegetación adyacente (Townsend, Arbuckle, Crow, \& Scarsbrook, 1997; Bojsen \& Jacobsen, 2003; Allan, 2004; Death \& Collier, 2010). En consecuencia, la pérdida de vegetación de ribera puede conducir a un menor ingreso de hojarasca y materia orgánica particulada a las fuentes de agua, y generar cambios en las características de rugosidad del lecho, la profundidad y velocidad del agua, lo cual incide en la disminución de hábitats y alimento para el ensamblaje de macroinvertebrados (Cummins, Wilzbach, Gates, Perry, \& Taliaferro, 1989) y por ende a cambios en su estructura y composición (Benstead, Douglas, \& Pringle, 2003; Bojsen \& Jacobsen, 2003; Wantzen \& Wagner, 2006).

A pesar de que se han realizado estudios sobre la diversidad taxonómica de macroinvertebrados acuáticos en corrientes tropicales, en su mayoría se limitan a describir los patrones y pocos intentan entender los mecanismos que los determinan (Ramírez \& Gutiérrez-Fonseca, 2014a). Algunos estudios se han dirigido a conocer la diversidad taxonómica y funcional en relación con los regímenes de caudal y disturbios hidrológicos, o a respuesta a factores ambientales y condiciones fisicoquímicas (e.g., Tomanova, Goitia, \& Helesic, 2006; RíosTouma, Encalada, \& Prat, 2011; Giraldo et al., 2014; Ríos-Pulgarín, Barletta, Arango-Jaramillo, \& Mancera-Rodríguez, 2016a; Tobes, Gaspar, Peláez, \& Miranda, 2016), pero existe poca información acerca de los efectos de la deforestación en las zonas de ribera sobre las comunidades de macroinvertebrados acuáticos (e.g. Bojsen \& Jacobsen, 2003).

Dado el aumento de la deforestación en la región Andina colombiana, la creciente pérdida de especies por actividades antrópicas y la falta de conocimiento de la respuesta taxonómica y funcional del ensamblaje de macroinvertebrados a dichas alteraciones, el objetivo de este trabajo fue establecer posibles efectos de la deforestación sobre los patrones de diversidad taxonómica y funcional del ensamblaje de macroinvertebrados acuáticos en cuatro quebradas Andinas colombianas, con y sin cobertura arbórea en sus márgenes. Se plantea como hipótesis de investigación que las estaciones sin cobertura arbórea presentarán disminución en la calidad de los hábitats, la diversidad biológica y las condiciones ambientales, por 
lo tanto, la diversidad taxonómica (estructura y composición) y la diversidad funcional (grupos funcionales tróficos y hábitos de vida) del ensamblaje de macroinvertebrados será menor en las estaciones con procesos de deforestación. Este estudio contribuirá a la generación de información útil para la formulación de estrategias efectivas de restauración y protección de bosques de ribera de quebradas Andinas y a la mitigación de los efectos negativos de las actividades agropecuarias sobre las zonas de margen de quebradas y cuerpos hídricos.

\section{MATERIALES Y MÉTODOS}

Área de estudio: El trabajo se realizó en las quebradas La Magdalena y Las Mercedes (afluentes del río Nare) y en las quebradas Peñoles y El Cardal (afluentes del río Guatapé), ubicadas en los municipios de Concepción, San Vicente, San Rafael y San Carlos, respectivamente, en el departamento de Antioquia, Colombia. El territorio es montañoso, con topografía ondulada, y las quebradas drenan hacia la cuenca media del río Magdalena, caracterizándose por ser torrenciales, de aguas muy claras y sin planicie aluvial (ManceraRodríguez, Castellanos-Barliza, \& UrregoBallestas, 2016). Los sitios se ubican entre los 898 msnm y 2140 msnm (Cuadro 1).

Se realizaron cuatro muestreos en los períodos de transición de lluvia a seco (junio de 2011), transición de seco a lluvia (septiembre 2011), lluvia (noviembre 2011) y seco (febrero de 2012). En cada una de las quebradas se seleccionaron tres sitios de muestreo para un total de 12 estaciones y se utilizó la clasificación de estaciones de referencia y alteradas establecida por Galeano-Rendón, MonsalveCortes \& Mancera-Rodríguez (2017), cada estación correspondió a un tramo de $100 \mathrm{~m}$. Estos autores definieron siete de las 12 estaciones evaluadas como estaciones de referencia ubicadas en las quebradas Las Mercedes (Estación E4), Peñoles (Estaciones E7, E8 y E9) y El Cardal (Estaciones E10, E11 y E12), las cuales presentan cobertura boscosa en sus márgenes sin alteraciones o con bajo grado de perturbación antrópica y cinco estaciones consideradas como alteradas, ubicadas en las quebradas La Magdalena (Estaciones E1, E2 y E3) y Las Mercedes (Estaciones E5 y E6), las cuales presentan alta perturbación antrópica, sin cobertura arbórea en sus márgenes, por actividades agrícolas y ganaderas (Cuadro 1).

Variables fisicoquímicas y caracterización ecológica: Se midieron las siguientes variables in situ: oxígeno disuelto (mg/L), saturación de oxígeno (\%), temperatura superficial del agua $\left({ }^{\circ} \mathrm{C}\right.$ ) con medidor Hanna (HI9828/401) y $\mathrm{pH}$, conductividad $(\mu \mathrm{S} / \mathrm{cm})$ y sólidos totales disueltos (STD) con medidor Hanna (9811-5). Se utilizaron los valores del Índice de calidad de la vegetación de ribera Andina (QBR-And), como principal indicador del nivel de pérdida de cobertura arbórea en los márgenes, el Índice de hábitat fluvial (IHF), y el Índice BMWP-Col, calculados para cada estación por Galeano et al. (2017). El ancho y la profundidad fueron medidos con flexómetro, y una vara graduada en $\mathrm{cm}$.

Recolecta e identificación de macroinvertebrados acuáticos: Los macroinvertebrados acuáticos se recolectaron usando captura manual sobre vegetación, restos de madera sumergida, rocas y áreas de deposición presentes en el cauce de las quebradas con la ayuda de pinzas entomológicas, y en las zonas con hojarasca y sedimento con red D-net de $405 \mathrm{~cm}^{2}$ y con malla de $500 \mu \mathrm{m}$, para lo cual se seleccionaron 10 puntos al azar a lo largo del tramo de $100 \mathrm{~m}$ de cada estación, según la metodología descrita por Roldán y Ramírez (2008). La identificación taxonómica se realizó mediante el uso de las claves de Posada y Roldán (2003), Manzo (2005), Domínguez, Molineri, Pescador, Hubbard y Nieto (2006), Silva-Passos, Nessimian, y Ferreira (2007), Merritt, Cummins y Berg (2008), Domínguez y Fernández (2009). Las muestras se preservaron y fueron depositadas en el Museo Entomológico Francisco Luis Gallego de la Universidad Nacional de Colombia Sede Medellín (código MEFLG). 


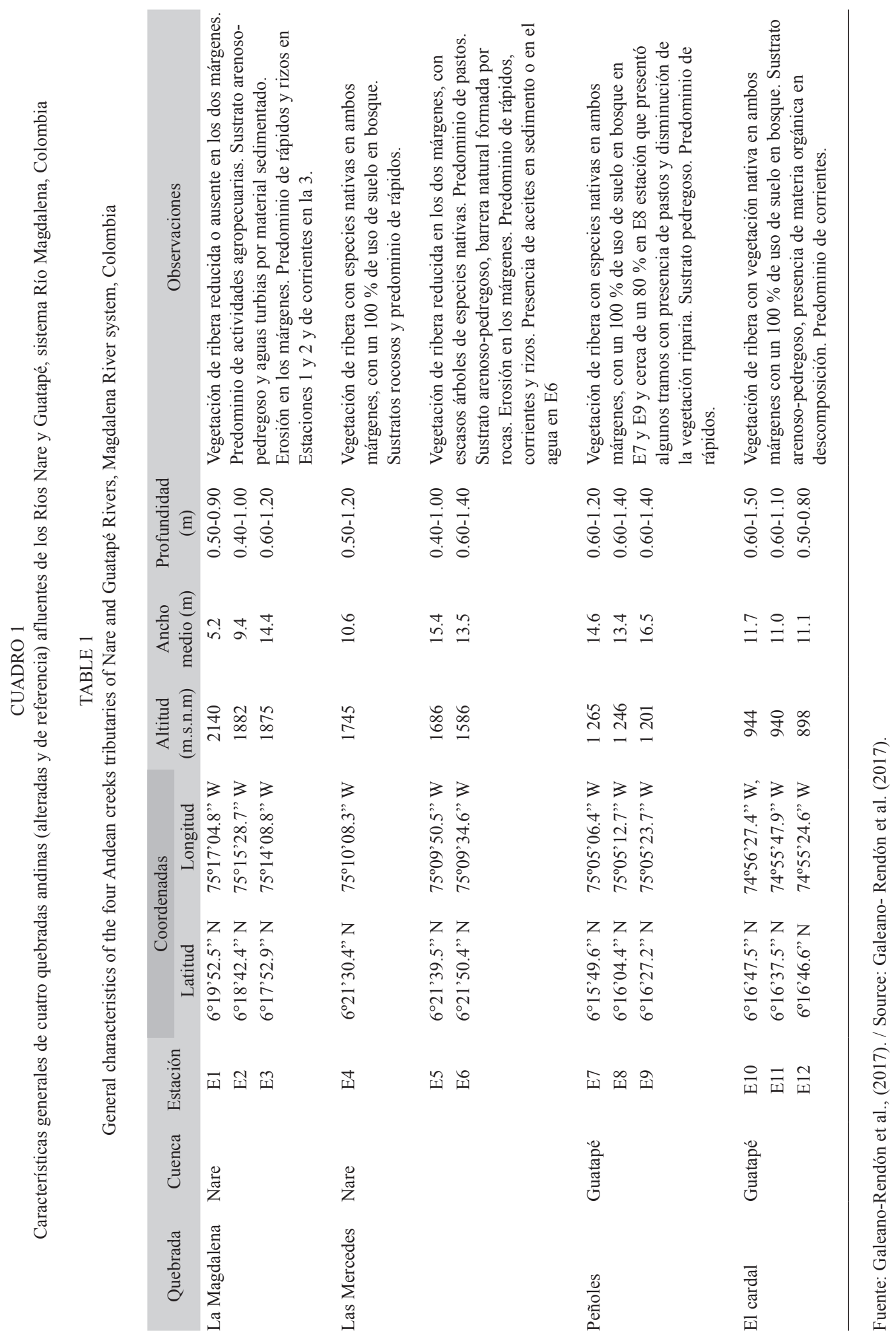


Análisis de datos: Para establecer si existieron diferencias significativas en los valores de las variables ambientales entre estaciones de referencia y alteradas, se aplicaron análisis de varianza unidireccionales (ANOVA) a cada variable, previa validación de los supuestos de normalidad (Kolmogorov-Smirnov) y homocedasticidad (prueba de Levene) y se realizaron comparaciones (post hoc) que fueron evaluadas mediante la prueba de Tukey para determinar los niveles que difieren en cada variable. Cuando los datos no cumplieron con los supuestos de normalidad y homocedasticidad (sensibilidad a los residuos del modelo) se utilizó la prueba no paramétrica de Kruskall-Wallis. También, se aplicaron ANOVAS para establecer diferencias significativas en la diversidad taxonómica y funcional entre estaciones de referencia y alteradas en general y por períodos hidrológicos.

Se calculó para las estaciones de referencia y alteradas el número de taxones e individuos, los índices de Shannon-Wienner, Dominancia, Equidad de Simpson, número de taxones raros (abundancia inferior al $3 \%$ ), utilizando el programa estadístico PAST (Hammer, Harper, \& Ryan, 2001). Los valores de los índices de diversidad Shannon-Weaver y dominancia de Simpson entre estaciones de referencia y alteradas se compararon mediante pruebas de t de Hutcheson (Zar, 1999). Igualmente, para determinar la condición ecológica se definió el número de taxones de Ephemeroptera, Plecóptera y Trichoptera (EPT). La diversidad funcional se evaluó mediante la asignación previa de los géneros que Ríos-Pulgarín et al. (2016a) dieron a los macroinvertebrados colectados en su estudio en los siguientes Grupos Funcionales Tróficos (GFT): colectores-filtradores $(\mathrm{CF})$, colectores-recolectores (CG), depredadores (PR), raspadores (SC) y fragmentadoresherbívoros $(\mathrm{SH})$; y en los siguientes hábitos de vida: excavadores $(\mathrm{Bu})$, escaladores $(\mathrm{Cb})$, reptadores $(\mathrm{Sp})$, nadadores $(\mathrm{Sw})$, buceadores (Di), agarradores $(\mathrm{Cg})$, rastreadores $(\mathrm{Cw}) \mathrm{y}$ patinadores (Sk).

Se definieron los patrones de variación en la composición taxonómica del ensamblaje de macroinvertebrados entre estaciones alteradas y de referencia con respecto a las variables ambientales y a los valores de los Índices IHF, QBR-And y BMWP-Col, mediante análisis discriminante canónico (ADC), que se realizó sobre datos de abundancia de los taxones que presentaron una frecuencia mayor al $5 \%$ y una abundancia superior a $3 \%$ (Gauch, 1982; RíosPulgarín et al., 2016a). En este análisis, los taxones que ayudan a explicar las diferencias se representan como vectores cuya longitud y dirección es proporcional a su contribución para explicar la variabilidad (Ríos-Pulgarín, Barletta, \& Mancera-Rodríguez, 2016b). Igualmente, se realizaron análisis discriminantes canónicos para evaluar la variabilidad de los diferentes grupos funcionales tróficos (GFT) y a los hábitos de vida del ensamblaje de macroinvertebrados entre las estaciones alteradas y de referencia. Todos los análisis se desarrollaron en versiones R Software 3.1.1 (R Core Team, 2012) y RWizard (Guisande, 2015).

\section{RESULTADOS}

Variables fisicoquímicas: Se encontraron diferencias significativas en el $\mathrm{pH}(\mathrm{P}<0.001)$ y la temperatura superficial del agua $(\mathrm{P}=0.014)$ entre los tipos de estaciones de estudio. El primer parámetro presentó un valor promedio mayor para las estaciones alteradas de 8.3, mientras que las estaciones de referencia fue de 7.8. Para el segundo parámetro, las estaciones de referencia presentaron mayor valor promedio de $18.7^{\circ} \mathrm{C}$, mientras que las alteradas tuvieron una media de 18.7 Las demás variables fisicoquímicas no presentaron diferencias significativas (Cuadro 2).

Variabilidad taxonómica de macroinvertebrados: Se identificaron 80 taxones y se capturaron un total de 8370 individuos de macroinvertebrados, de los cuales 1612 individuos de 50 taxones fueron recolectados en las estaciones alteradas y 6758 individuos de 75 taxones en las estaciones de referencia. El orden más abundante tanto en las estaciones alteradas como en las de referencia fue Trichoptera (37.2\% y $41.0 \%$, respectivamente), 
CUADRO 2

Valores de los índices y características químicas de cuatro quebradas andinas (alteradas y de referencia) afluentes de los Ríos Nare y Guatapé, sistema Río Magdalena, Colombia. Colombia

TABLE 2

Values of the indexes and chemical characteristics of altered and reference stations in four Andean streams, tributaries of Nare and Guatapé Rivers, Magdalena River system, Colombia

\begin{tabular}{lcccccc} 
& \multicolumn{3}{c}{ Estaciones alteradas (5) } & \multicolumn{3}{c}{ Estaciones de referencia (7) } \\
& Media & D.S (Min-Max) & C.V \% & Media & D.S (Min-Max) & C.V \% \\
IHF & 50.6 & $8.7(37-61)$ & 17.2 & 79.7 & $7.8(70-97)$ & 9.8 \\
QBR-And & 15.0 & $8.4(5-25)$ & 55.8 & 82.9 & $8.8(70-95)$ & 10.6 \\
$\mathrm{BMWP} / \mathrm{Col}$ & 60.3 & $18.1(36-84)$ & 30.0 & 107.4 & $6.9(98-119)$ & 6.4 \\
$\mathrm{O}_{2}(\mathrm{mg} / \mathrm{L})$ & 9.2 & $1.9(7-12)$ & 20.5 & 10.0 & $2.2(7.3-14.4)$ & 22.5 \\
$\mathrm{pH}$ & 8.3 & $0.3(8-9)$ & 3.8 & 7.8 & $0.2(7.5-8.0)$ & 2.0 \\
Conductividad $(\mu \mathrm{S} \mathrm{cm}-1)$ & 24.3 & $2.5(21-28)$ & 10.2 & 26.2 & $8.2(16.3-40.3)$ & 31.2 \\
Temperatura agua $\left({ }^{\circ} \mathrm{C}\right)$ & 18.7 & $1.0(18-20)$ & 5.2 & 19.8 & $1.5(17-22)$ & 7.5 \\
Saturación de Oxigeno $(\%)$ & 83.7 & $31.7(30-110)$ & 37.8 & 93.6 & $27.2(67-151)$ & 29.0 \\
T.S.D (mg/L) & 20.5 & $4.9(16-28)$ & 24.0 & 25.4 & $9.6(14.3-42.3)$ & 37.8 \\
\hline
\end{tabular}

Fuente: Galeano et al. (2017).Source: Galeano et al. (2017).

Desviación Estandar (D.S), Coeficiente de Variación (C.V) y Solidos Disueltos Totales (T.D.S).

por otro lado, en las estaciones alteradas se presentó un porcentaje significativamente mayor de los órdenes Ephemeroptera (27.4\%) (P = $0.017)$, y Diptera (12.3\%) $(\mathrm{P}=0.024)$, mientras que en las estaciones de referencia fueron significativamente mayores Hemiptera (15.9 $\%)(\mathrm{P}<0.001)$ y Coleoptera $(14.7 \%)(\mathrm{P}=$ 0.003 ) (Fig. 1A).

En cuanto a los períodos hidrológicos, en las estaciones alteradas no se encontró una variación significativa en la abundancia relativa de macroinvertebrados con un valor máximo en el muestreo de transición de seco a lluvia de $30 \%$ y un mínimo en la época de transición de lluvia-seco de $21.1 \%$. Para las mismas estaciones, en todos los períodos de muestreo el orden más abundante fue Trichoptera, con excepción de la transición lluvia-seco (19.2\%) donde se observó una diferencia significativa respecto al período de lluvias $(48.2 \%)(\mathrm{P}=0.020)$. Los órdenes Ephemeroptera y Díptera presentaron un comportamiento opuesto a Trichoptera con incremento de su abundancia en los períodos de transición y con una disminución marcada durante el período de lluvia. Para las estaciones de referencia, se presentó variación en la abundancia relativa de macroinvertebrados entre períodos hidrológicos, con un valor máximo en el muestreo de época seca $(36.2 \%)$ y un mínimo en el período de lluvia (12.2\%). Trichoptera presentó los valores más altos en la época seca y en las transiciones disminuyó en el período de lluvias, a su vez durante el período de transición de seco a lluvia Hemiptera disminuyó y presentó diferencias significativamente con los otros períodos hidrológicos $(\mathrm{P}$ $<0.03$ ), además Ephemeroptera incrementó su abundancia relativa $(\mathrm{P}=0.011)$ (Fig. 1B).

Los valores de riqueza, taxones raros, el número medio de taxones EPT y el índice de Shannon-Weaver fueron mayores para las estaciones de referencia, pero sólo se presentaron diferencias estadísticas significativas para el índice de Shannon-Weaver $(\mathrm{P}<0.001)$. La dominancia presentó valores bajos, y la equidad de Simpson presentó diferencia significativa $(p=0.039)$ entre estaciones alteradas y de referencia (Cuadro 3 ).

Para las estaciones de referencia, los taxones Grumichella, Rhagovelia, Helicopsyche, Cylloepus, Smicridea, Anacroneuria, Thraulodes, Baetodes, Simulium y Leptonema 

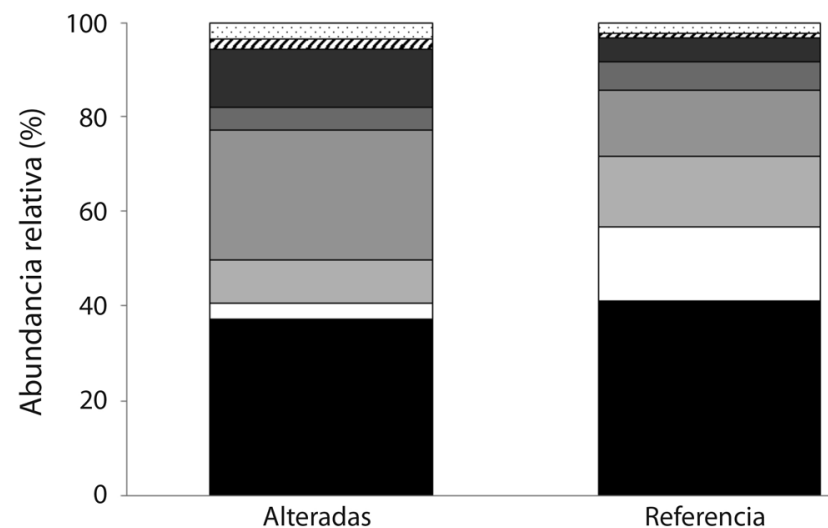

๑Otros

A

ZOdonata

口iptera

口Plecoptera

$\square$ Ephemeroptera

$\square$ Coleoptera

$\square$ Hemiptera

-Trichoptera

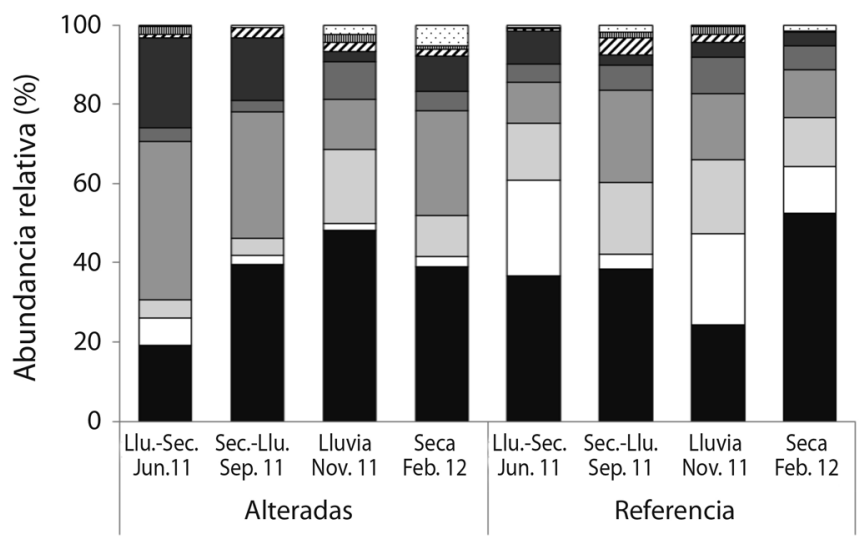

Tipo de estación

Fig. 1. Abundancia relativa de órdenes de macroinvertebrados en estaciones alteradas y de referencia (A), y para los cuatro períodos hidrológicos (B) en cuatro quebradas Andinas afluentes de los Ríos Nare y Guatapé, sistema Río Magdalena, Colombia.

Fig. 1. Relative abundance of macroinvertebrate orders in altered and reference stations (A), and for the four hydrological periods (B) in four Andean creeks tributaries of Nare and Guatapé Rivers, Magdalena River system, Colombia.

representaron el $79.4 \%$ de la abundancia relativa, y para las estaciones alteradas los taxones Leptonema, Baetodes, Simulium, Smicridea, Baetis, Anacroneuria, Thraulodes, Cylloepus, Moribaetis y Mortoniella representaron el 70.5 $\%$. El análisis canónico discriminante separó las estaciones alteradas y de referencia y representó el $100 \%$ de la varianza en el primer eje canónico. En las estaciones alteradas, los taxones que contribuyeron a la variación fueron Leptonema, Baetodes, Moribaetis y Mayobaetis, que se asociaron con los mayores valores de $\mathrm{pH}$, y altura sobre el nivel del mar (ASNM), mientras que Thraulodes, Anacroneuria, Rhagovelia, Helicopsyche, Macrelmis, Grumichella, Cylloepus y Smicridea se asociaron a las estaciones de referencia y a los mayores valores del índice de calidad de bosque de ribera Andina (QBR-And), el índice de hábitat fluvial (IHF) y el Índice BMWP, así como con la temperatura del agua, el oxígeno disuelto (OD) y la profundidad (Fig. 2).

Variabilidad funcional: En las estaciones alteradas los grupos funcionales tróficos (GFT) que dominaron en abundancia fueron 


\section{CUADRO 3}

Valores de diversidad y condición ecológica para el ciclo hidrológico anual de cuatro quebradas Andinas (alteradas y de referencia) afluentes de los Ríos Nare y Guatapé, sistema Río Magdalena, Colombia

TABLE 3

Values of diversity, ecological conditions for altered and reference stations for the hydrological cycle

\begin{tabular}{|c|c|c|c|c|c|c|}
\hline & \multicolumn{3}{|c|}{ Alteradas } & \multicolumn{3}{|c|}{ Referencia } \\
\hline & Mínimo & Máximo & Media & Mínimo & Máximo & Media \\
\hline \multicolumn{7}{|l|}{ Diversidad } \\
\hline Número de taxones & 14 & 35 & 25 & 35 & 42 & 39 \\
\hline Número de individuos & 162 & 457 & 322.4 & 390 & 1231.0 & 965.4 \\
\hline Índice de Shannon $\left(\mathrm{H}^{\prime}\right)$ & 2.17 & 2.70 & 2.41 & 2.02 & 3.03 & 2.58 \\
\hline Dominancia (D) & 0.12 & 0.17 & 0.14 & 0.07 & 0.23 & 0.13 \\
\hline Equidad de Simpson (1-D) & 0.83 & 0.88 & 0.86 & 0.77 & 0.93 & 0.87 \\
\hline Número de taxones raros & 6 & 23 & 13 & 27 & 35 & 31 \\
\hline \multicolumn{7}{|l|}{ Condición ecológica } \\
\hline Taxones EPT & 8 & 13 & 10.8 & 14 & 15 & 14.9 \\
\hline
\end{tabular}

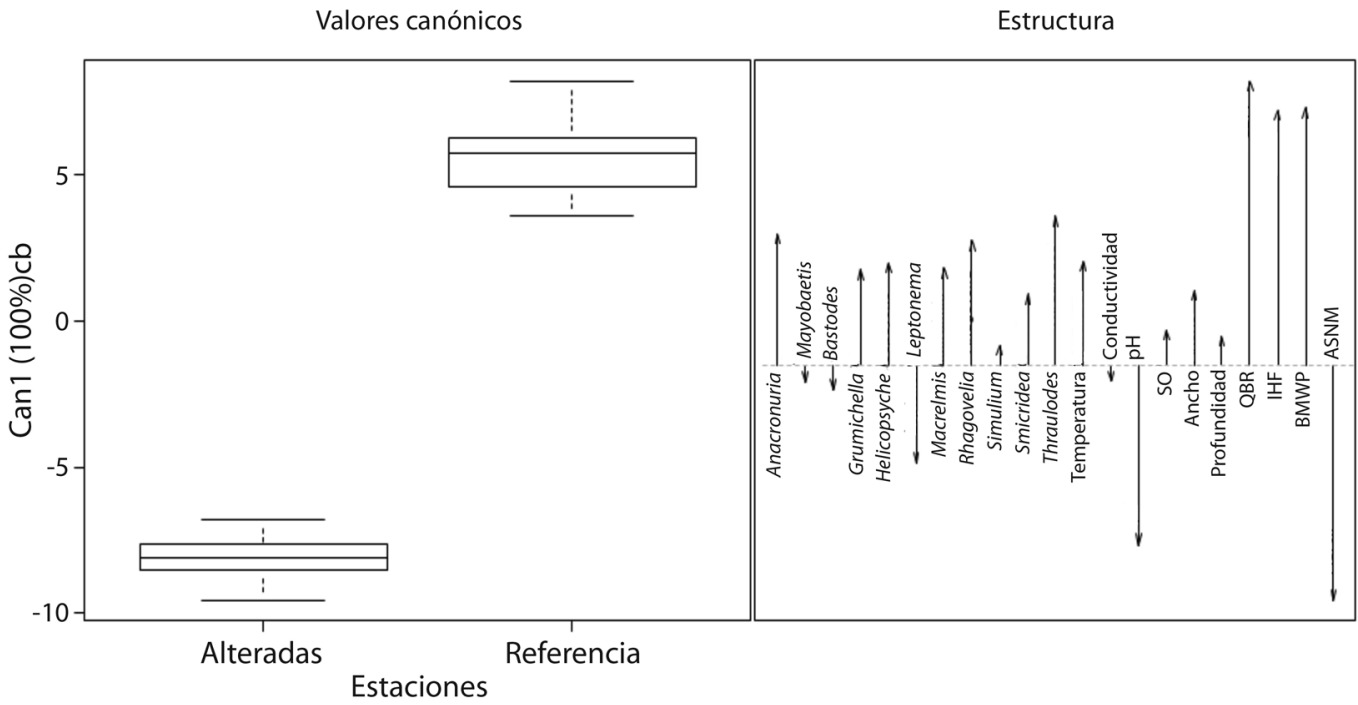

Fig. 2. Análisis discriminante canónico entre estaciones alteradas y referencia, basado en la abundancia de macroinvertebrados y variables ambientales en cuatro quebradas Andinas afluentes de los Ríos Nare y Guatapé, sistema Río Magdalena, Colombia. SO, saturación de oxígeno; QBR, calidad de bosque de ribera; IHF, índice de hábitat fluvial; BWMP, Índice Biological Monitoring Working Party y ASNM, altura sobre el nivel del mar.

Fig. 2. Discriminant canonical analysis between altered seasons and reference, based on macroinvertebrates abundance and environmental variables in four Andean creeks tributaries of Nare and Guatapé Rivers, Magdalena River system, Colombia. SO, oxygen saturation; QBR, quality of riverside forest; IHF, river habitat index; BWMP, Biological Monitoring Working Party Index ASNM, elevation above sea level.

colectores-filtradores (CF) $(27.5 \%)$, colectores- recolectores/raspadores $(\mathrm{CG} / \mathrm{SC})(17.4$ $\%)$, raspadores $(\mathrm{SC})(15.7 \%)$ y colectoresrecolectores (CG) $(14 \%)$, mientras que para las estaciones de referencia los GFT más abundantes fueron colectores-recolectores/raspadores $(\mathrm{CG} / \mathrm{SC})(27.2 \%)$, raspadores $(\mathrm{SC})$ (25.4\%) y depredadores (PR) (24\%) (Fig. 3A). Se encontraron diferencia significativa entre las estaciones para los grupos funcionales tróficos 

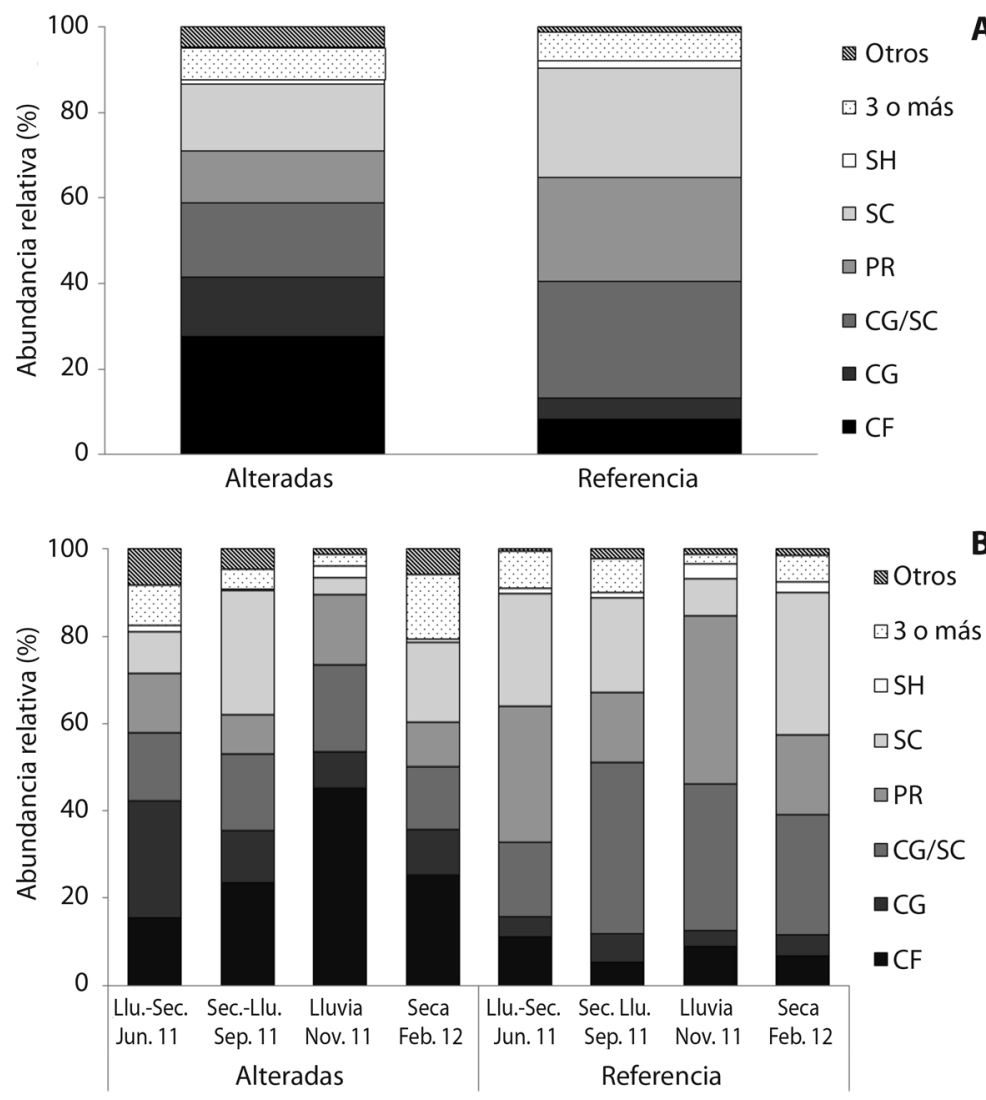

Tipo de estación

Fig. 3. Abundancia relativa de grupos funcionales tróficos (GFT) de macroinvertebrados en las estaciones alteradas y de referencia (A), y para los cuatro períodos hidrológicos (B) en cuatro quebradas Andinas afluentes de los Ríos Nare y Guatapé, sistema Río Magdalena, Colombia. CG, colectores-recolectores; CF, colectores-filtradores; SH, fragmentadoresherbívoros; SC, raspadores; PR, depredadores.

Fig. 3. Relative abundance of functional feeding groups (FFG) of macroinvertebrates in altered and reference stations (A), and for the four hydrological periods in four Andean creeks tributaries of Nare and Guatapé Rivers, Magdalena River system, Colombia. CG, collectors-gatherers; CF, collectors-filters; SH, shredders-herbivores; SC, scrapers; PR, predators.

$\mathrm{CF}(\mathrm{P}=0.013)$ y colectores-recolectores $\mathrm{CG}(\mathrm{P}$ $=0.004)$ que presentaron valores mayores en estaciones alteradas y en $\mathrm{CG} / \mathrm{SC}(\mathrm{P}<0.001)$ y $\mathrm{PR}(\mathrm{P}=0.001)$ que presentaron valores mayores en estaciones de referencia (Fig. 3A).

En las estaciones alteradas el GFT colectores-filtradores (CF) presentó diferencias significativas entre los períodos de transición en los que tuvo menor abundancia, con relación a los períodos de lluvia $(\mathrm{P}=0.010)$ y $\operatorname{seco}(\mathrm{P}=$ 0.020). El GFT raspadores (SC) se incrementó durante el período de transición de seco a lluvia y presentó diferencia significativa con el período de lluvias en el cual su abundancia relativa disminuyó significativamente $(\mathrm{P}=0.021)$ (Fig. 3B). En las estaciones de referencia el GFT más abundante fue colectores-recolectores/raspadores $(\mathrm{CG} / \mathrm{SC})$ en todos los muestreos, con excepción del período transición de lluvia a seca en el cual disminuyó su abundancia y presentó diferencias significativas con el período de transición de seco a lluvia $(\mathrm{P}=0.021)$. Aunque los demás GFT no presentaron diferencias significativas entre períodos hidrológicos, se 
observó un incremento de depredadores (PR) en el período de lluvia y de raspadores (SC) en el período seco (Fig. 3B).

El hábito de vida más abundante tanto en las estaciones alteradas como en las de referencia fue agarradores $(\mathrm{Cg})(68.1 \%$ y $50.5 \%$, respectivamente), pero en las estaciones alteradas se presentó un porcentaje significativamente mayor de los hábitos excavadores $(\mathrm{Bu})$ y nadadores $(\mathrm{Sw})(\mathrm{P}=0.02)$, mientras que en las estaciones de referencia fueron significativamente mayores los patinadores (Sk) (0.01) y los taxones con tres o más hábitos $(\mathrm{P}=0.04)$ (Fig. 4A). Por períodos hidrológicos para las estaciones alteradas el hábito de vida más abundantes fue agarradores $(\mathrm{Cg})$ en todos los períodos y se observó un incremento de taxones con tres o más hábitos durante los dos períodos de transición y de excavadores $(\mathrm{Bu})$ en la transición lluvia-seca y en el período seco, aunque sin diferencias significativas. Para las estaciones de referencia el hábito de vida agarradores $(\mathrm{Cg})$
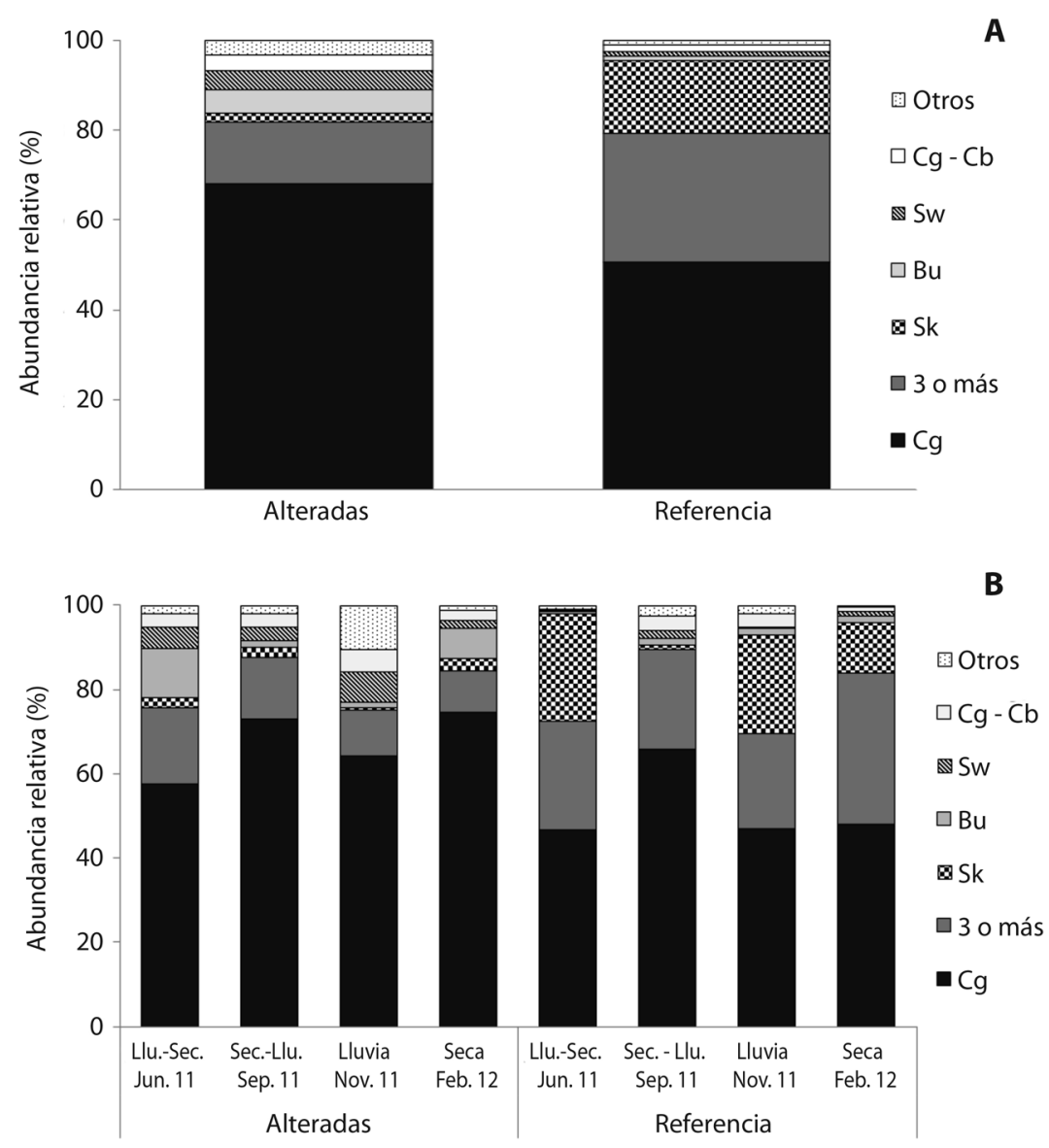

Tipo de estación

Fig. 4. Abundancia relativa de hábitos de vida de macroinvertebrados en las estaciones alteradas y de referencia (A), y para los cuatro períodos hidrológicos (B) en cuatro quebradas Andinas afluentes de los Ríos Nare y Guatapé, sistema Río Magdalena, Colombia. Bu, excavadores; Cb, escaladores; Sp, reptadores; Sw, nadadores; Di, buceadores; Cg, agarradores; $\mathrm{Cw}$, rastreadores; $\mathrm{Sk}$, patinadores.

Fig. 4. Relative abundance of life habits macroinvertebrates in altered and reference stations (A), and for the four hydrological periods in four Andean creeks tributaries of Nare and Guatapé Rivers, Magdalena River system, Colombia. (B). $\mathrm{Bu}$, burrowers; $\mathrm{Cb}$, climbers; Sp, sprawlers; Sw, swimmers; Di, divers; Cg, clingers; Cw, crawlers; Sk, skaters. 
presentó los valores más altos durante todos los períodos hidrológicos, aunque su mayor abundancia se presentó en el período de transición de seco a lluvia, coincidiendo con una disminución significativa en ese período del hábito patinadores Sk $(\mathrm{P}<0.01)$ (Fig. 4B).

El hábito de vida más abundante tanto en las estaciones alteradas como en las de referencia fue agarradores $(\mathrm{Cg})(68.1$ y $50.5 \%$, respectivamente). Sin embargo, en las estaciones alteradas se presentó un porcentaje significativamente mayor de los hábitos excavadores $(\mathrm{Bu})$ y nadadores $(\mathrm{Sw})(\mathrm{P}=0.02)$, mientras que en las estaciones de referencia fueron significativamente mayores los patinadores (Sk) (0.01) y los taxones con tres o más hábitos $(\mathrm{P}=0.04)$ (Fig. 4A). En todos los períodos hidrológicos, para las estaciones alteradas el hábito de vida más abundantes fue agarradores $(\mathrm{Cg})$, y se observó, para los dos períodos de transición, un incremento de taxones con tres o más hábitos, y en la transición lluvia-seco y seco de excavadores $(\mathrm{Bu})$, aunque no significativamente diferente. Para las estaciones de referencia el hábito de vida agarradores $(\mathrm{Cg})$ presentó los valores más altos durante todos los períodos hidrológicos, aunque su mayor abundancia se presentó en el período de transición de seco a lluvia, coincidiendo con una disminución significativa en ese período del hábito patinadores Sk $(\mathrm{P}<0.01)$ (Fig. 4B).

El análisis canónico discriminante aplicado a los GFT y a los hábitos de vida separó los dos tipos de estaciones evaluadas y representó el $100 \%$ de la varianza en el primer eje canónico. Las estaciones alteradas estuvieron asociadas con el GFT colectores-filtradores (CF) y con mayores valores de $\mathrm{pH}$, mientras que las estaciones de referencia se asociaron con los GFT colectores-recolectores/raspadores $(\mathrm{CG} / \mathrm{SC})$, depredadores (PR), raspadores (SC) y fragmentadores-herbívoros (SH) y con mayores valores de QBR, IHF, BMWP y temperatura del agua (Fig. 5A). Con relación a los hábitos de vida, las estaciones alteradas estuvieron asociadas con escaladores/nadadores/buceadores $(\mathrm{Cb} / \mathrm{Sw} / \mathrm{Di})$, escaladores $(\mathrm{Cb})$, excavadores $(\mathrm{Bu})$ y nadadores $(\mathrm{Sw})$ y con valores mayores de $\mathrm{pH}$ y corresponden a estaciones ubicadas a mayor altura sobre el nivel del mar (ASNM). Por su parte, las estaciones de referencia se asociaron con hábitos de vida agarradores $(\mathrm{Cg})$, patinadores $(\mathrm{Sk})$, reptadores (Sp) y con taxones con tres hábitos como agarradores/rastreadores/reptadores $(\mathrm{Cg} / \mathrm{Cw} / \mathrm{Sp})$ y agarradores/nadadores/buceadores $(\mathrm{Cg} / \mathrm{Sw} / \mathrm{Di})$ y con mayores valores de QBR, IHF, BMWP y temperatura (Fig. 5B).

\section{DISCUSIÓN}

Las diferencias encontradas en la temperatura del agua (mayor en estaciones de referencia), está asociada con el nivel altitudinal, ya que las estaciones alteradas están ubicadas en promedio en zonas de mayor altura sobre el nivel del mar y por ende presentan menor temperatura, por lo que no fue posible atribuir efectos sobre esta variable de la pérdida de cobertura arbórea en los márgenes. Sin embargo, mayores temperaturas del agua se han asociado con la deforestación debido a la disminución de la sombra y al consiguiente aumento de la insolación solar (e.g. Dodds, 2002; Chapman \& Chapman, 2003). Al respecto, Lorion y Kennedy (2009) encontraron en cuerpos de agua con márgenes cubiertos por pastos temperaturas del agua significativamente más altas que las de los bosques y sugieren que el sombreado ribereño es un factor importante en la regulación de la temperatura, además Jacobsen, Schultz, y Encalada (1997) encontraron que la riqueza de insectos incrementó de manera lineal con el aumento de la temperatura en quebradas tropicales. Por otra parte, el mayor valor promedio del $\mathrm{pH}$ en las estaciones alteradas, puede estar relacionado con cambios en el uso de suelo como consecuencia de las diferentes actividades agropecuarias. Esto coincide con lo reportado por Mesa (2010) quien encontró mayores valores de $\mathrm{pH}$ en áreas afectadas por uso agrícola y ganadero correspondientes a sitios degradados por la deforestación en quebradas Andinas en Argentina, por $\mathrm{Bu}$, Meng, Zhang y Wan (2014) quienes mencionan que el pH presentó 

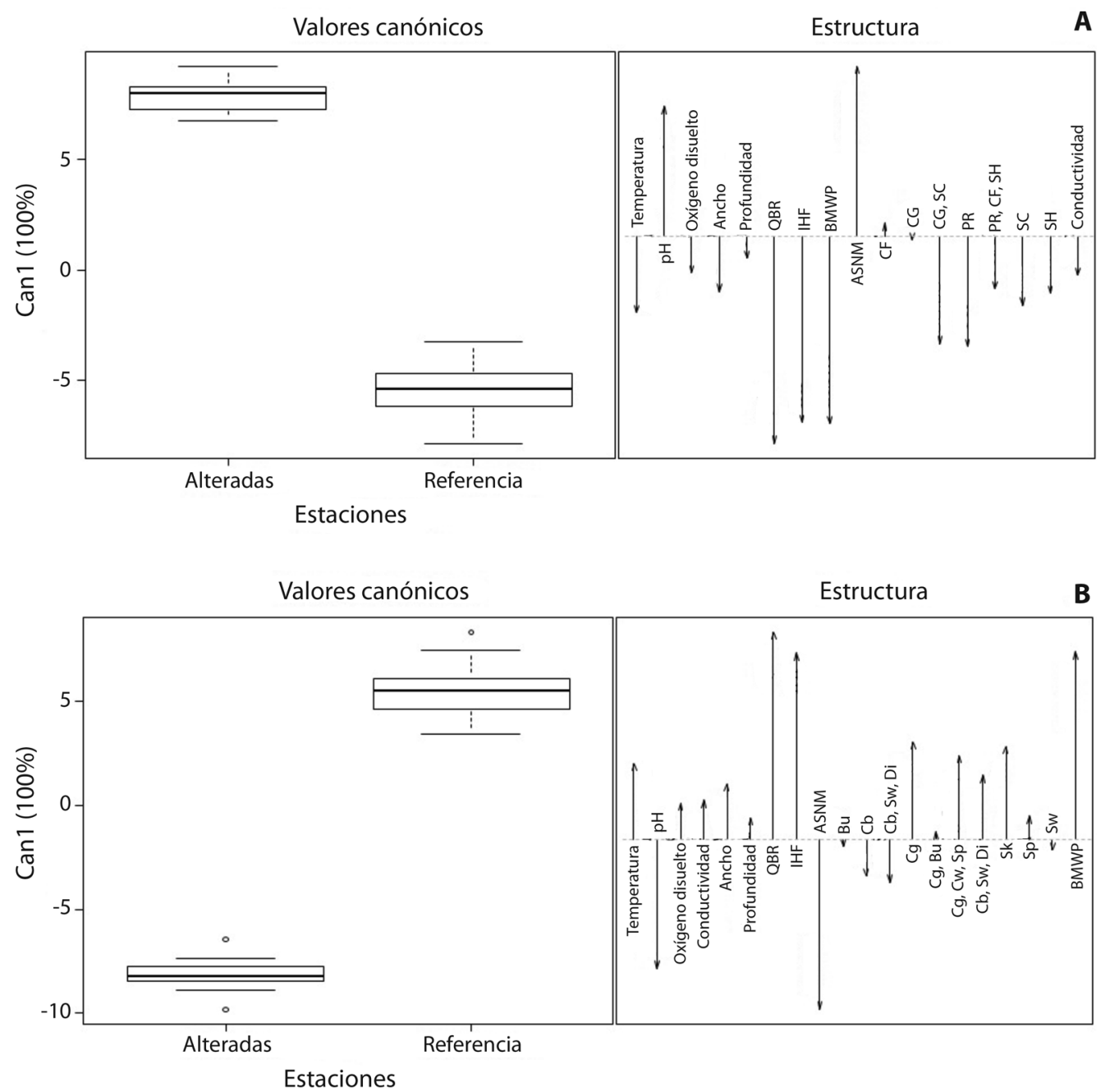

Fig. 5. (A) Análisis discriminante canónico (ADC) entre estaciones alteradas y referencia basado en grupos funcionales tróficos (GFT) de macroinvertebrados y variables ambientales, y (B) ADC entre estaciones alteradas y referencia basado en hábitos de vida de macroinvertebrados y variables ambientales en cuatro quebradas Andinas afluentes de los Ríos Nare y Guatapé, sistema Río Magdalena, Colombia. CG, colectores-recolectores; CF, colectores-filtradores; SH, fragmentadoresherbívoros; SC, raspadores; PR, depredadores. Bu, excavadores; $\mathrm{Cb}$, escaladores; Sp, reptadores; Sw, nadadores; Di, buceadores; $\mathrm{Cg}$, agarradores; $\mathrm{Cw}$, rastreadores; $\mathrm{Sk}$, patinadores.

Fig. 5. (A) Canonical discriminant analysis (CDA) between altered seasons and reference, based on functional feeding groups (FFG) of macroinvertebrates and environmental variables, and (B) CDA between altered seasons and reference, based on life habits of macroinvertebrates and environmental variables in four Andean creeks tributaries of Nare and Guatapé Rivers, Magdalena River system, Colombia. CG, collectors-gatherers; CF, collectors-filters; SH, shreddersherbivores; SC, scrapers; PR, predators. Bu, burrowers; Cb, climbers; Sp, sprawlers; Sw, swimmers; Di, divers; Cg, clingers; $\mathrm{Cw}$, crawlers; Sk, skaters.

una correlación negativa con la proporción de cobertura arbórea y un aumento de sus valores en zonas con usos de suelo agrícola sobre los márgenes en ríos en China y por Kaushal et al. (2018) que menciona que el aumento de sales con bases fuertes y carbonatos eleva el $\mathrm{pH}$ en los ecosistemas sin bosques ribereños.

El ensamblaje de macroinvertebrados en las estaciones de referencia presentó mayor abundancia, diversidad y número de taxones. 
Esto coincide con estudios que han encontrado que la diversidad y riqueza de especies disminuye en áreas que han sido impactadas por actividades humanas como la deforestación (e.g. Trayler \& Davis, 1998; Benstead et al., 2003; Mesa 2010). Bojsen y Jacobsen (2003) encontraron que la diversidad fue menor en los sitios deforestados y la composición de macroinvertebrados en sitios boscosos fue más heterogénea, además, Couceiro, Hamada, Luz, Forsberg, y Pimentel (2007) encontraron que la mayor deforestación se correlacionó con una menor riqueza de taxones, aunque en su estudio ésta no afectó la abundancia. Además, la modificación de bosques de ribera por cambios de uso del suelo en áreas de pastos y cultivos puede llevar a la disminución de material alóctono que ingresa al cuerpo hídrico y a la alteración de la calidad del hábitat con la menor disponibilidad de sustratos aportados por los bosques adyacentes (e.g. Uieda \& Kikuchi, 1995; Bücker, Sondermann, Frede, \& Breuer, 2010; Giraldo et al. 2014), el aumento en la carga de sedimentos que disminuye la heterogeneidad del hábitat (Allan, Erickson, \& Fay, 1997) y afecta la capacidad de las especies para tolerar cambios en los recursos alimenticios (Benstead \& Pringle, 2004). Lo anterior, indica una relación directa entre la deforestación y la disminución de diversidad taxonómica, debido a que la reducción de elementos disponibles como los sustratos o el material alóctono homogeniza el hábitat impidiendo el establecimiento de alimento y refugios para las especies (Schlösser, 1991). Además, la cobertura arbórea evita la entrada de sedimentos, contaminantes y nutrientes que puedan llegar directamente al cauce (Gergel, Turner, Miller, Melack, \& Stanley, 2002; Palma, Figueroa, \& Ruiz, 2009).

Los órdenes más abundantes en el estudio fueron Trichoptera y Ephemeroptera, lo cual coincide con lo reportado para otras quebradas Andinas por Posada, Roldan y Ramírez (2000) y por Mathuriau, Thomas, y Chauvet (2008), pero difiere de lo reportado por Giraldo et al. (2014) y por Ríos-Pulgarín et al. (2016a) que mencionan como orden más abundante a Diptera, y de Torres-Zambrano y Torres-Zambrano
(2016) que encontraron más abundancia de los órdenes Coleoptera y Diptera por encima de Ephemeroptera y Trichoptera.

El orden Trichoptera en sistemas lóticos se ha asociado con una alta cobertura vegetal en sus márgenes, siendo importantes eslabones en la transferencia de energía dentro de estos ecosistemas (Oliveira \& Bispo, 2001) y se ha documentado la disminución o desaparición de poblaciones de este orden en zonas deforestadas (Benstead et al., 2003; Lorion \& Kennedy, 2009). En las estaciones alteradas se encontró una mayor abundancia del orden Díptera (e.g. Chironomidae y Simulidae), la cual se puede asociar con su forma de alimentación filtradora que le permite beneficiarse de los sólidos en suspensión, además de que prefieren flujos de agua continua y de mayor velocidad (Gallardo-Mayenco \& Toja, 2002), Esto también fue reportado por Giraldo et al. (2014) quienes encontraron mayor abundancia de Díptera en estaciones con intervención antrópica con ganadería. Por otra parte, la mayor abundancia del orden Ephemeroptera coincide con lo reportado por Bücker et al. (2010) para quebradas de montaña en el sur de Ecuador, que encontraron mayor abundancia de este orden en estaciones que estaban ubicadas en áreas deforestadas, en comparación con las áreas boscosas. Se presentó mayor abundancia de Hemiptera y Coleoptera en las estaciones de referencia, lo que se relaciona en el caso de Hemiptera con sus hábitos depredadores (Rhagovelia) debido a una mayor cantidad de refugios que pueden usar para capturar sus presas, y en el caso de Coleoptera con una mayor disponibilidad de materia orgánica gruesa que es fuente de alimentación para fragmentadores (Anchytarsus), particularmente aquella que ha sido colonizada por hongos o bacterias, permitiendo que los macroinvertebrados asimilen en corto tiempo el alimento (Cummins \& Klug, 1979).

Los cambios en la estructura del ensamblaje de macroinvertebrados en corrientes Neotropicales, también ocurren de acuerdo a la época hidrológica del año (Bojsen \& Jacobsen, 2003). En las estaciones alteradas, los órdenes Ephemeroptera y Díptera se incrementaron en 
los períodos de transición y tuvieron una disminución marcada durante el período de lluvia, lo cual puede deberse a una mayor entrada de sedimentos al cauce y a un mayor arrastre. Por su parte, la mayor abundancia relativa de macroinvertebrados para las estaciones de referencia se dio en época seca, presentando el orden Trichoptera los valores más altos en dicho período, lo cual coincide con lo reportado por Mathuriau et al. (2008) que menciona que el descenso del nivel del agua permite la construcción de refugios para especies de este orden. Al respecto, se conoce que la disponibilidad del sustrato está relacionada con el mantenimiento de la vegetación riparia (Bojsen $\&$ Barriga, 2002) y que su presencia se asocia con mayor cantidad de refugios contra los depredadores y para soportar las corrientes de agua. También parece haber una relación con la disponibilidad de alimento, la construcción de casas de Trichoptera, y la presencia de sitios de ovoposición y emergencia para los insectos acuáticos (Couceiro et al., 2007). El aumento en la abundancia del ensamblaje de macroinvertebrados en el período seco también ha sido reportado en otros estudios de corrientes Andinas no degradadas (Flecker \& Feifarek, 1994; Jacobsen \& Encalada, 1998; Ramírez, Pringle \& Douglas, 2006; Ríos-Pulgarín et al., 2016a).

La menor abundancia relativa se presentó en la época de lluvias, que corresponde a los períodos donde se pueden presentar mayores cambios en las variables ambientales por el aumento del caudal y la velocidad de la corriente, así como por el ingreso de más sedimentos al cauce. En el caso de los insectos acuáticos en ambientes lóticos, el aumento del caudal en el período lluvioso puede incrementar del arrastre de los organismos, disminuyendo la abundancia de los mismos (Oliveira \& Bispo, 2001).

Las estaciones de referencia presentaron mayor promedio de taxones EPT, debido a que dichos ordenes son sensibles a las perturbaciones antrópicas, por lo que suelen estar más asociados a quebradas con zonas ribereñas protegidas que presentan mejores condiciones de calidad del agua y de hábitat que en las quebradas con influencia de ganadería (e.g.
Moya, Oberdorff, Bigorne, Zubieta \& Camacho, 2006; Lorion \& Kennedy, 2009; Death \& Collier, 2010; Giraldo et al., 2014). Los cambios en la riqueza de los taxones EPT asociados con la alteración de la vegetación ribereña se han relacionado con las especies de macroinvertebrados que dependen de la alimentación de materia alóctona (Death \& Collier, 2010; Burcher, Valett, \& Benfield, 2007). El porcentaje de cobertura boscosa influye en la proporción de taxones EPT, ya que estos ordenes desempeñan un papel importante en el ciclo de los nutrientes y la transferencia de energía (Ferreira et al., 2014; Graca, 2001; Castro, Sylvain, \& Callisto, 2017).

En las estaciones alteradas fueron importantes los taxones Leptonema, Baetodes y Mayobaetis, lo que coincide con lo reportado por (Lorion \& Kennedy, 2009), que encontraron que la familia Baetidae fue más abundante en corrientes dominadas por matrices de pastizales, lo cual se debe a que son taxones que no dependen del carbono terrestre directamente sino que pueden aprovechar el aumento de la producción primaria de los arroyos. Lo anterior, se debe a la disminución en la salud en las corrientes deforestadas cuando la productividad primaria excede sustancialmente la respiración (Bunn, Davies, \& Mosisch, 1999). Zuñiga, Rojas, y Mosquera (1997) mencionan que Baetodes se caracteriza por ser un organismo adaptado a diferentes tipos de calidad de agua. Por su parte, a las estaciones de referencia se asociaron los taxones Thraulodes, Anacroneuria, Rhagovelia, Helicopsyche, y Macrelmis, con mayores valores de los índices QBR-And, IHF y BMWP, menor temperatura del agua y mayor oxígeno disuelto. Una mayor abundancia de Thraulodes se ha asociado a zonas de bosque (Lorion \& Kennedy, 2009), ya que es altamente sensible a disminuciones de oxígeno y a condiciones de contaminación (Zuñiga et al., 1997); además Macrelmis ha sido reportado como característico de zonas no degradadas (Mesa, 2010).

Los grupos funcionales tróficos estuvieron dominados por recolectores y raspadores, por taxones generalistas con dos o más grupos 
tróficos, similar a lo reportado por Ramírez y Pringle (1998), Tomanova et al. (2006) y Ríos-Pulgarín et al. (2016a). Sin embargo, los GFT mostraron cambios relacionados con la presencia o ausencia de vegetación de ribera en las estaciones. En las estaciones alteradas se encontró mayor abundancia de colectoresrecolectores (CG) y de colectores-filtradores (CF). Lo cual coincide con lo encontrado por Bojsen y Jacobsen (2003) quienes mencionan que la densidad relativa de colectores y filtradores aumentó en quebradas con mayor grado de deforestación, y por Ramírez \& GutiérrezFonseca (2014b) que destacan que los CG son abundantes en las áreas con flujo lento donde las partículas finas son abundantes y los CF capturan partículas directamente de la columna de agua como en el caso de estaciones alteradas por deforestación donde se presenta mayor ingreso de sedimentos.

Por su parte, para las estaciones de referencia los GFT que presentaron diferencias en abundancia fueron colectores-recolectores/ raspadores $(\mathrm{CG} / \mathrm{SC})$ y depredadores $(\mathrm{PR})$. La mayor abundancia de colectores-recolectores/ raspadores se ha relacionado con la rápida y continua descomposición del material alóctono en detritus finos que ocurre en arroyos tropicales durante el ciclo hidrológico anual (Dobson, Magana, Mathooko, \& Ndegwa, 2002; Tomanova et al., 2006). La presencia de grupos tróficos raspadores y depredadores en estaciones de referencia está asociada con la mayor disponibilidad de hábitats y mayor movilidad en los diferentes sustratos, sin embargo, esto implica mayor exposición al flujo y por ende más riesgo de deriva (Tomanova et al., 2006). La mayor abundancia relativa de PR en estaciones con cobertura arbórea en sus márgenes, coincide con lo encontrado por Bojsen y Jacobsen (2003) quienes mencionan que la densidad relativa de depredadores aumentó con una mayor cobertura del dosel y la cantidad de detritus de la hojarasca. Otros estudios en ríos Andinos tropicales con cobertura boscosa mencionan como GFT más abundante el de colectores, seguido por los fragmentadores y los depredadores (e.g. Ríos-Touma, Encalada,
\& Prat, 2009; Chará-Serna, Chará, Zúñiga, Pedraza, \& Giraldo, 2010; Chará-Serna, Chará, Zúñiga, Pearson, \& Boyero, 2012).

La presencia de CG/SC y taxones con dos o más GFT en estaciones de referencia y alteradas puede estar más relacionada con alta disponibilidad de hábitats, principalmente en estaciones con cobertura de bosque en sus márgenes, y a una relativa flexibilidad trófica que reduce la superposición de nichos que disminuye la competencia inter e intraespecífica (Woodward \& Hildrew, 2002), lo que contribuye a incrementar la supervivencia y facilitar la colonización espacial de los insectos acuáticos (Tomanova et al., 2006; Ríos-Touma et al. 2011). Esta flexibilidad trófica es usual en ecosistemas loticos tropicales ya que, probablemente le facilita a la comunidad de macroinvertebrados colonizar espacial y temporalmente diferentes hábitats (Mihuc, 1997).

El GFT fragmentadores-herbívoros (SH) tuvo una representación menor en las estaciones estudiadas, lo cual es contrario a lo reportado para otras quebradas tropicales por Ríos-Touma et al., (2009) y Chará-Serna et al. (2010; 2012), quienes resaltan que la dominancia de colectores y fragmentadores reflejan la importancia de la hojarasca como recurso alimentario para la entomofauna. Sin embargo, otros estudios también han encontrado baja abundancia de SH (e.g. Dobson et al. 2002; Rueda-Delgado, Wantzen, \& Tolosa, 2006; Wantzen \& Wagner, 2006), y se ha asociado esto con la acelerada descomposición de la hojarasca por microorganismos debido a las altas temperaturas y a que organismos grandes, como camarones y peces, podrían reemplazar los fragmentadores en quebradas tropicales (Landeiro, Hamada, \& Melo, 2008). A pesar de la baja abundancia de SH se presentó una asociación positiva de éste GFT con las estaciones de referencia, particularmente del Anchytarsus de la familia Ptilodactylidae, se ha asociado con la abundancia de detritus de la hojarasca (Bojsen \& Jacobsen, 2003). Igualmente, CharáSerna et al. (2010), sugiere que los fragmentadores pueden ser más importantes en términos de biomasa por su tamaño y pueden presentar 
una alta importancia ecológica a pesar de la baja abundancia.

Con relación a los hábitos de vida, no existe información disponible sobre su respuesta a procesos de deforestación en otros sistemas. El hábito de vida predominante, tanto en estaciones alteradas como de referencia, fue el de los agarradores $(\mathrm{Cg})$, que está asociado a macroinvertebrados que presentan adaptaciones morfológicas que les permiten adherirse a los sustratos cuando las corrientes son rápidas (Hanson, Springer, \& Ramírez, 2010; RíosPulgarín et al., 2016a). Ríos Pulgarín et al. (2016a) también reportan un predominio de hábitos agarradores en un río Andino colombiano y lo interpretan como una estrategia de adaptación a los frecuentes eventos de arrastre. La presencia de hábitos excavadores $(\mathrm{Bu})$ es mayor en las estaciones alteradas en transición de lluvias a seco y en época seca, lo cual está asociado a que estos macroinvertebrados se suelen enterrar en sedimentos blandos (Hanson et al., 2010) y lo hacen como respuesta adaptativa a las condiciones de sequía (Ríos-Pulgarín et al., 2016a). Los nadadores ( $\mathrm{Sw}$ ) se asociaron más a estaciones alteradas principalmente en el período de lluvias, lo que coincide con lo reportado por (Ríos-Pulgarín et al., 2016a), quienes encontraron que los individuos con este hábito se asociaron con períodos de gran caudal durante años de mayor lluvia producidos por el fenómeno de La Niña. Por su parte, los patinadores (Sk) y reptadores $(\mathrm{Sp})$ se asociaron más a estaciones de referencia, lo que puede deberse a que dichos organismos se arrastran en los sustratos disponibles como hojarasca o madera (Hanson et al., 2010), los cuales son más abundantes en las estaciones con vegetación riparia. Estos resultados, pueden estar relacionados al escenario ecológico-evolutivo en el que los macroinvertebrados de las corrientes han evolucionado y la estrecha relación con la perturbación, la dureza ambiental y la presión humana (Díaz, Suarez, \& Vidal-Abarca, 2008).

En conclusión, la deforestación condiciona la diversidad taxonómica y funcional del ensamblaje de macroinvertebrados. Las estaciones alteradas presentaron mayor abundancia de los órdenes Díptera y Ephemeroptera, menor número de familias $\mathrm{y}$ de individuos, menor diversidad de Shannon y mayor abundancia de la familia Baetidae (Mayobaetis, Baetidae) y del género Leptonema. Por su parte, las estaciones de referencia presentaron mayor presencia de los órdenes Coleóptera y Hemíptera, mayor número de familias, $\mathrm{y}$ de individuos, mayor diversidad de Shannon, así como mayor abundancia de la familia Leptophlebiidae (Thraulodes) y del género Macrelmis. Desde el punto de vista funcional, las estaciones alteradas presentaron mayor abundancia de los GFT colectoresrecolectores (CG) y de colectores-filtradores (CF) y de los hábitos de vida excavadores $(\mathrm{Bu})$ y nadadores $(\mathrm{Sw})$, mientras que las estaciones de referencia presentaron predominancia de los GFT colectores-recolectores/raspadores (CG/ $\mathrm{SC})$ y depredadores (PR) y el hábito de vida patinador. Con base en lo anterior, se estableció que la diversidad taxonómica (estructura y composición) del ensamblaje de macroinvertebrados fue menor en estaciones con procesos de deforestación y a su vez la diversidad funcional presentó cambios importantes en la composición y abundancia de grupos funcionales tróficos y los hábitos de vida con presencia de taxones más generalistas y con adaptaciones a ambientes con mayor alteración en las estaciones sin cobertura arbórea en sus márgenes, lo que permite aceptar la hipótesis de investigación.

Así mismo, es importante resaltar que el conocimiento de las variables que inciden sobre la estructura taxonómica y funcional de los ensamblajes de macroinvertebrados, y cómo éstos responden a afectaciones a la deforestación, puede ayudar a determinar la escala a la cual se deben realizar los esfuerzos de protección o restauración que mejoren o mantengan las condiciones ambientales y ecológicas de los ríos. También es necesario aumentar el conocimiento biológico de los hábitos de vida $\mathrm{y}$ de los grupos funcionales tróficos y su respuesta a estas afectaciones, de manera que se puedan usar como indicadores de conservación de los recursos hídricos y bosques de los bosques de ribera Andina. 
Declaración de ética: los autores declaran que todos están de acuerdo con esta publicación y que han hecho aportes que justifican su autoría; que no hay conflicto de interés de cualquier tipo; y que han cumplido con todos los requisitos y procedimientos éticos y legales pertinentes. El documento firmado se encuentra en los archivos de la revista.

\section{AGRADECIMIENTOS}

A la empresa ISAGEN S.A. E.S.P., a la Corporación Autónoma Regional de los Ríos Negro y Nare-CORNARE y a la Universidad Nacional de Colombia por la financiación del proyecto 20101009235 "Estudio de la biología, ecología y diversidad genética de las poblaciones naturales de sabaleta Brycon henni en las cuencas de los ríos Nare y Guatapé, Antioquia”.

\section{RESUMEN}

Las quebradas están fuertemente influenciadas por su vegetación ribereña y la deforestación afecta a la comunidad acuática. Se estudiaron los efectos de la deforestación sobre la diversidad taxonómica y funcional del ensamblaje de macroinvertebrados acuáticos en cuatro quebradas Andinas colombianas. El estudio incluyó 12 sitios, siete de ellos con cobertura arbórea en sus márgenes en áreas boscosas no perturbadas por la deforestación (estaciones de referencia) y los otros cinco sin cobertura arbórea en áreas deforestadas (estaciones alteradas). Se realizaron cuatro muestreos de campo entre junio 2011 y febrero 2012 y se recolectaron muestras de macroinvertebrados bentónicos utilizando el método D-net con malla de 500 $\mu \mathrm{m}$ y recolección manual, y se midieron diferentes variables físicoquímicas in situ. Se calcularon los índices de Shannon-Wienner (H') y Simpson (D). Se aplicaron análisis unidireccionales de varianza ANOVAs a cada variable ambiental y análisis canónicos discriminantes (CDA). Se recolectaron un total 8370 individuos correspondientes a 80 taxones durante el estudio. La abundancia y diversidad fue mayor en las estaciones localizadas en áreas boscosas no perturbadas. Thraulodes, Anacroneuria y Rhagovelia, se asociaron con valores más altos de Calidad de Bosque de Ribera Andina (QBR-And), Índice de Hábitat Fluvial (IHF), Biological Monitoring Working Party Colombia (BMWP/Col) y temperatura en estaciones de referencia, mientras que Leptonema, Baetodes y Mayobaetis se asociaron con mayores valores de $\mathrm{pH}$ y altura sobre nivel del mar en estaciones alteradas. Los principales grupos funcionales tróficos (GFT) en las estaciones de referencia fueron colectores-recolectores/raspadores (27.2\%), raspadores $(25.4 \%)$ y depredadores $(24.4 \%)$, mientras que en estaciones alteradas fueron: Colectores filtradores (27.5\%), colectores-recolectores/raspadores (17.4\%) у raspadores $(15.7 \%)$. Los hábitos de vida más abundantes en las estaciones de referencia fueron agarradores (50.5 $\%)$, taxones con tres o más hábitos $(28.7 \%)$ y patinadores $(16.1 \%)$, mientras que en las estaciones alteradas fueron agarradores (68.1\%), taxones con tres o más hábitos (13.7 $\%$ ) y excavadores $(5.2 \%)$. El conocimiento de las variables que determinan la estructura taxonómica y funcional de los ensamblajes de macroinvertebrados, y cómo responden a impactos como la deforestación, puede ayudar a determinar acciones de protección o restauración de las condiciones ambientales y ecológicas de las quebradas Andinas y los hábitats riparios.

Palabras clave: diversidad taxonómica; grupos funcionales tróficos (GFT); hábitos de vida.

\section{REFERENCIAS}

Allan, J. D. (2004). Landscapes and riverscapes: the influence of land use on stream ecosystems. Annual Review of Ecology Evolution and Systematics, 35, 257-284.

Allan, D. J., Erickson, D. L., \& Fay, J. (1997). The influence of catchment land use on stream integrity across multiple spatial scales. Freshwater Biology, 37, 149-161.

Armenteras, D., Cabrera, E., Rodríguez, N., \& Retana, J. (2013). National and regional determinants of tropical deforestation in Colombia. Regional Environmental Change, 13, 1181-1193.

Armenteras, D., Rodríguez, N., Retana, J., \& Morales, M. (2011). Understanding deforestation in montane and lowland forests of the Colombian Andes. Regional Environmental Change, 11(3), 693-705.

Benstead, J., Douglas, M., \& Pringle, C. (2003). Relationships of Stream Invertebrate Communities to Deforestation in Eastern Madagascar. Ecological Applications, 13, 1473-1490.

Benstead, J. D., \& Pringle, C. M. (2004). Deforestation alters the resource base and biomass of endemic stream insects in eastern Madagascar. Freshwater Biology, 49, 490-501.

Bojsen, B. H., \& Barriga, R. (2002). Effects of deforestation on fish community structure in Ecuadorian Amazon streams. Freshwater Biology, 47, 2246-2260.

Bojsen, B. H., \& Jacobsen, D. (2003). Effects of deforestation on macroinvertebrate diversity and assemblage structure in Ecuadorian Amazon streams. Archiv für Hydrobiologie, 158, 317-342. 
Bu, H., Meng, W., Zhang, Y., \& Wan, J. (2014). Relationships between land use patterns and water quality in the Taizi River basin, China. Ecologycal Indicadors, 41, 187-197.

Bücker, A., Sondermann, M., Frede, H. G., \& Breuer, L. (2010). The influence of land-use on macroinvertebrate communities in montane tropical streams a case study from Ecuador. Fundamental and Applied Limnology, 177, 267-282.

Burcher, C. L., Valett, H. M., \& Benfield, E. F. (2007). The land-cover cascade: relationships coupling land and water. Ecology, 88, 228-242.

Bunn, S., Davies, P., \& Mosisch, T. (1999). Ecosystem measures of river health and their response to riparian and catchment degradation. Freshwater Biology, 41, 333-345.

Castro, D., Sylvain, D., \& Callisto, M. (2017). Landscape variables influence taxonomic and trait composition of insect assemblages in Neotropical savanna streams. Freshwater Biology, 62, 1472-1486.

Chapman, C. A., \& Chapman, L. J. (2003). Deforestation in tropical Africa: impacts on aquatic ecosystems. In T. L. Crisman, L. J. Chapman, C. A. Chapman, \& L. S. Kaufman (Eds.), Conservation, Ecology and Management of African Freshwaters (pp. 229-246). Florida, Gainesville: University Press of Florida, Gainesville.

Chará-Serna, A. M., Chará, J. D., Zúñiga, M. C., Pearson, R. G., \& Boyero, L. (2012) Diets of leaf-litter-associated insects in three Colombian streams. Annales de Limnologie - International Journal of Limnology, 48, 139-144.

Chará-Serna, A. M., Chará, J. D., Zúñiga, M. C., Pedraza, G., \& Giraldo, L. (2010). Clasificación trófica de insectos acuáticos en ocho quebradas protegidas de la ecorregión cafetera colombiana. Universitas Scientiarum, 15(1), 27-36.

Couceiro, N., Hamada, N., Luz, S., Forsberg, B., \& Pimentel, P. (2007). Deforestation and sewage effects on aquatic macroinvertebrates in urban streams in Manaus, Amazonas, Brazil. Hydrobiologia, 575, 271-284.

Cummins, K., \& Klug, M. (1979). Feeding ecology of stream invertebrates. Annual Review of Ecology, Evolution and Systematics, 10, 147-72.

Cummins, K. W., Wilzbach, M. A., Gates, D. M., Perry, J. B., \& Taliaferro, W. B. (1989). Shredders and riparian vegetation. Bioscience, 39, 24-30.

Death, R. G. (1996). The effect of patch disturbance on stream invertebrate community structure: the influence of disturbance history. Oecologia, 108, 567-576.
Death, R. G., \& Collier, K. J. (2010). Measuring stream macroinvertebrate responses to gradients of vegetation cover: when is enough enough? Freshwater Biology, 55, 1447-1464.

Death, R. G., \& Winterbourn, M. J. (1995). Diversity patterns in stream benthic invertebrate communities: the influence of habitat stability. Ecology, 76, 1446-1460.

Diaz, A., Suarez, M., \& Vidal-Abarca, M. (2008). Biological traits of stream macroinvertebrates from a semi-arid catchment: patterns along complex environmental gradients. Freshwater Biology, 53, 1-21.

Dobson, M., Magana, A., Mathooko, J. M., \& Ndegwa, F. K. (2002). Detritivores in Kenyan highland streams: more evidence for the paucity of shredders in the tropics? Freshwater Biology, 47, 909-919.

Dodds, W. K. (2002). Freshwater Ecology, Concepts and Environmental Applications. San Diego, USA: Academic Press Inc.

Domínguez, E., \& Fernández, H. R. (2009). Macroinvertebrados bentónicos sudamericanos. Sistemática y Biología. Tucumán, Argentina: Fundación Miguel Lillo.

Domínguez, E., Molineri, C., Pescador, M. L., Hubbard, M. D., \& Nieto, C. (2006). Ephemeroptera of South America. In J. Adis, J. R. Arias, G. Rueda-Delgado, \& K. M. Wantzen (Eds.), Aquatic Biodiversity in Latin America (ABLA) (Vol. 2, pp. 1-646). SofiaMoscow: Pensoft.

Ferreira, W. R., Ligeiro, R., Macedo, D. R., Hughes, R. M., Kaufmann, P. R., \& Oliveira, L. G. (2014). Importance of environmental factors for the richness and distribution of benthic macroinvertebrates in tropical headwater streams. Freshwater Science, 33(3), 860-871.

Flecker, A. S., \& Feifarek, B. (1994). Disturbance and the temporal variability of invertebrate assemblages in two Andean streams. Freshwater Biology, 31,131-142.

Galeano-Rendón, E., Monsalve-Cortes, L., \& ManceraRodríguez, N. J. (2017). Evaluación de la calidad ecológica de quebradas Andinas en la cuenca del Rio Magdalena, Colombia. Revista U.D.C.A Actualidad \& Divulgación Científica, 20(2), 413-424.

Gallardo-Mayenco, A., \& Toja, J. (2002). Spatio-temporal distribution of Simuliids (Diptera) and associated environmental factors in two mediterranean basins of Southern Spain. Limnetica, 21(1), 47-57.

Gauch, H. G. (1982). Multivariate analysis in community ecology. Cambridge, England: Cambridge University Press.

Gergel, S. E., Turner, M. G., Miller, J. R., Melack, J. M., \& Stanley, E. H. (2002). Landscape indicators of 
human impacts to riverine systems. Aquatic Sciences, 64, 118-128.

Giraldo, L., Chará, J., Zúñiga, M., Chará-Serna, A., \& Pedraza, G. (2014). Impacto del uso del suelo agropecuario sobre macroinvertebrados acuáticos en pequeñas quebradas de la cuenca del río La Vieja (Valle del Cauca, Colombia). Revista de Biología Tropical, 62(Suppl. 2), 203-219.

Graca, M. A. (2001). The role of invertebrates on leaf litter decomposition in streams - A review. International Review of Hydrobiology, 86, 383-393.

Guisande, C. (2015). StatR. Retrieved from http://www. ipez.es/RWizard

Hammer, Ø., Harper, D. А. T., \& Ryan, P. D. (2001). PAST: Paleontological Statistics Software Package for Education and Data Analysis. Palaeontología Electrónica. Retrieved from http://palaeoelectronica. org/2001_1/past/issue1_01.htm

Hanson, P., Springer, M., \& Ramírez, A. (2010). Introducción a los grupos de macroinvertebrados acuáticos. Revista Biología Tropical, 58(Suppl. 4), 3-37.

Jacobsen, D., \& Encalada, A. (1998). The Macroinvertebrate fauna of Ecuadorian high-land streams in the wet and dry season. Archiv fur Hydrobiologie, 142(1), 53-70.

Jacobsen, D., Schultz, R., \& Encalada, A. (1997). Structure and diversity of stream invertebrate assemblages: the influence of temperature with altitude and latitude. Freshwater Biology, 38, 247-261.

Kaushal, S., Likens, G., Pace, M., Utz, R., Hag, S., Gorman, J., \& Grese, M. (2018). Freshwater salinization syndrome on a continental scale. Proceedings of the National Academy of Science. DOI: 10.1073/ pnas. 1711234115

Landeiro, V. L., Hamada, N., \& Melo, A. S. (2008). Responses of aquatic invertebrate assemblages and leaf breakdown to macroconsumer exclusion in Amazonian "terra firme" streams. Fundamental and Applied Limnology, 172(1), 49-58.

Lorion, C. M., \& Kennedy, B. P. (2009). Riparian forest buffers mitigate the effects of deforestation on fish assemblages in tropical headwater streams. Ecological Applications, 19(2), 468-479.

Mancera-Rodríguez, N., Castellanos-Barliza, J., \& UrregoBallestas, D. (2016). Biología reproductiva de Saccodon dariensis (Teleostei: Parodontidae) en afluentes del río Guatapé, cuenca del río Magdalena, Colombia. Revista de Biología Tropical, 64(2), 635-653.

Manzo, V. (2005). Key to the South America genera of Elmidae (Insecta: Coleoptera) with distributional data. Studies on Neotropical Fauna and Environment, 40(3), 201-208.
Mathuriau, C., Thomas, G. B., \& Chauvet, E. (2008). Seasonal dynamics of benthic detritus and associated macroinvertebrate communities in a Neotropical stream. Fundamental and Applied Limnology, 171(4), 323-333.

Merritt, R., Cummins, K., \& Berg, M. (2008). An introduction to the aquatic insects of North America. Iowa, U.S.A.: Kendall Hunt Publishing Company.

Mesa, L. M. (2010). Effect of spates and land use on macroinvertebrate community in Neotropical Andean streams. Hydrobiologia, 641, 85-95.

Mihuc, T. B. (1997). The functional trophic role of lotic primary consumers: generalist versus specialist strategies. Freshwater Biology, 37, 455-462.

Moya, N., Oberdorff, T., Bigorne, R., Zubieta, J., \& Camacho, J. (2006). Comunidad de macroinvertebrados en ríos de la cuenca Chipiriri con condiciones de referencia y alteradas, estudio preliminar para la bioevaluación de arroyos. Ciencia abierta Internacional, 30, 1-27.

Oliveira, L. G., \& Bispo, P. C. (2001). Ecologia de comunidades das larvas de Trichoptera Kirby (Insecta) em dois córregos de primeira ordem da Serra dos Pirineus, Pirenópolis, Goiás, Brasil. Revista Brasileira de Zoologia, 18(4), 1245-1252.

Palma, A., Figueroa, R., \& Ruiz, V. (2009). Evaluación de ribera y hábitat fluvial a través de los índices QBR e IHF. Gayana, 73(1), 57-63.

Posada, J., \& Roldán, G. (2003). Clave ilustrada y diversidad de las larvas de Trichoptera en el Noroccidente de Colombia. Caldasia, 25(1), 169-192.

Posada, J. A., Roldán, G., \& Ramírez, J. J. (2000). Caracterización físicoquímica y biológica de la calidad de aguas de la cuenca de la quebrada Piedras Blancas, Antioquia, Colombia. Revista Biología Tropical, 48, 59-70.

R Core Team. (2012). $R$ : A language and environment for statistical computing. R Foundation for Statistical Computing. Vienna, Austria. Retrieved from https:// www.R-project.org/

Ramírez, A., \& Gutiérrez-Fonseca, P. (2014a). Estudios sobre macroinvertebrados acuáticos en América Latina: Avances recientes y direcciones futuras. Revista de Biología Tropical, 62(2), 9-20.

Ramírez, A., \& Gutiérrez-Fonseca, P. (2014b). Functional feeding groups of aquatic insect families in Latin America: a critical analysis and review of existing literature. Revista de Biología Tropical, 62(2), 155-167.

Ramírez, A., \& Pringle, C. M. (1998). Structure and production of a benthic insect assemblage in a 
Neotropical stream. Journal of the North American Benthological Society, 17, 443-463.

Ramírez, A., Pringle, C. M., \& Douglas, M. (2006). Temporal and spatial patterns in stream physicochemistry and insect assemblages in tropical lowland streams. The North American Benthological Society, 25(1), 108-125.

Ríos-Pulgarín, M. I., Barletta, M., Arango-Jaramillo, M. C., \& Mancera-Rodríguez, N. J. (2016a). The role of hydrological cycle on the patterns of macroinvertebrate assemblages in an Andean piedmont stream in Colombia. Journal of Limnology, 75(1), 107-120.

Ríos-Pulgarín, M. I., Barletta, M., \& Mancera-Rodríguez, N. J. (2016b). The role of the hydrological cycle on the distribution patterns of fish assemblages in an Andean stream. Journal of Fish Biology, 89(1), 102-130.

Ríos-Touma, B., Encalada, A., \& Prat, N. (2009). Leaf litter dynamics and its use by invertebrates in a highaltitud tropical Andean stream. International Review of Hydrobiology, 94(4), 357-371.

Ríos-Touma, B., Encalada, A., \& Prat, N. (2011). Macroinvertebrate assemblages of an Andean high altitude tropical stream: the importance of season and flow. International Review of Hydrobiology, 96(6), 667-685.

Roldán, G., \& Ramírez, J. J. (2008). Fundamentos de limnología neotropical. Medellín, Colombia: Editorial Universidad de Antioquia.

Rueda-Delgado, G. K., Wantzen, M., \& Tolosa, M. B. (2006). Leaf-Litter decomposition in an Amazonian floodplain stream: effects of seasonal hydrological changes. Journal of the North American Benthological Society, 25, 233-249.

Schlösser, I. J. (1991). Stream fish ecology: a landscape perspective. BioScience, 41, 704-712.

Schneider, K. N., \& Winemiller, K. O. (2008). Structural complexity of woody debris patches influences fish and macroinvertebrate species richness in a temperate floodplain river system. Hydrobiologia, 610, 235-244.

Silva-Passos, M., Nessimian, J., \& Ferreira, N. (2007). Chaves para identificação dos gêneros de Elmidae (Coleoptera) ocorrentes no Estado do Rio de Janeiro, Brasil. Revista Brasileira de Entomología, 51(1), $42-53$.

Sweeney, B. W., Bott, T. L., Jackson, J. K., Kaplan, L. A., Newbold, J. D., Standley, L. J., ... Horwitz, R. J.
(2004). Riparian deforestation, stream narrowing, and loss of stream ecosystem services. Proceedings of the National Academy of Sciences of the United States of America, 101, 14132-14137.

Tobes, I., Gaspar, S., Peláez, R., \& Miranda, M. (2016). Spatial distribution patterns of fish assemblages relative to macroinvertebrates and environmental conditions in Andean piedmont streams of the Colombian Amazon. Inland Waters, 6(1), 89-104.

Torres-Zambrano, N. N., \& Torres-Zambrano, D. R. (2016). Macroinvertebrados acuáticos de la quebrada los Alisos, Firavitoba - Boyacá. Intropica, 11, 47-56.

Tomanova, S., Goitia, E., \& Helešic, J. (2006). Trophic levels and functional feeding groups of macroinvertebrates in Neotropical streams. Hydrobiologia, 556, 251-264.

Townsend, C. R., Arbuckle, C. J., Crowl, T. A., \& Scarsbrook, M. R. (1997). The relationship between land-use and physicochemistry, food resources and macroinvertebrate communities in tributaries of the Tarieri River, New Zealand: a hierarchically scaled approach. Freshwater Biology, 37, 177-191.

Trayler, K. M., \& Davis, J. A. (1998). Forestry impacts and the vertical distribution of stream invertebrates in south-west Australia. Freshwater Biology, 40, 331-342.

Uieda, V. S., \& Kikuchi, R. M. (1995). Entrada de material aloctone (detritos e invertebrados terrestres) num pequeno curso de a'gua corrente na cuesta de Botucatu, Sao Paulo. Acta Liminologica Brasiliensia, 7, 105-114.

Wantzen, K. M., \& Wagner, R. (2006). Detritus processing by invertebrate shredders: a neotropical-temperate comparison. Journal of the North American Benthological Society, 25, 216-232.

Woodward, G., \& Hildrew, A. G. (2002). Body-size determinants of niche overlap and intraguild predation within a complex food web. Journal of Animal Ecology, 71, 1063-1074.

Zar, J. H. (1999). Biostatistical Analysis. New Jersey, USA: Prentice-Hall.

Zuñiga, M. C., Rojas, A. M., \& Mosquera, S. (1997). Biological aspects of Ephemeroptera in rivers of Southwestern Colombia (South America). In P. Landolt \& M. Sartori (Eds.), Ephemeroptera and Plecoptera: Biology, Ecology, Systematics (pp. 261-268). Fribourg, Switzerland: MTL - Mauron + Tinguely \& Lachat SA. 